\title{
Correction to: SEOM Clinical Guideline for treatment of muscle-invasive and metastatic urothelial bladder cancer (2016)
}

\author{
M. Lázaro ${ }^{1} \cdot$ E. Gallardo ${ }^{2} \cdot$ M. Doménech ${ }^{3} \cdot$ Á. Pinto ${ }^{4} \cdot$ A. González-del-Alba ${ }^{5} \cdot$ J. Puente $^{6} \cdot$ O. Fernández $^{7} \cdot$ A. Font $^{8}$. \\ N. Lainez ${ }^{9} \cdot$ S. Vázquez ${ }^{10}$
}

Published online: 26 February 2019

(c) Federación de Sociedades Españolas de Oncología (FESEO) 2019

\section{Correction to: Clin Transl Oncol (2016) 18:1 197-1205 https://doi.org/10.1007/s12094-016-1584-z}

Due to a technical issue, the family name of the author, A. González del Alba, was displayed incorrectly in PubMed. The correct family name should read as González-del-Alba, A.

\author{
M. Lázaro \\ martin.lazaro.quintela@sergas.es \\ E. Gallardo \\ egallardo@tauli.cat \\ M. Doménech \\ mdomenech@althaia.cat \\ Á. Pinto \\ alvaropintomarin@gmail.es \\ A. González-del-Alba \\ aranzazu.gonzalezdelalba@ssib.es \\ J. Puente \\ docjavip@yahoo.es \\ O. Fernández \\ ovidio.fernandez.calvo@sergas.es

\section{A. Font} \\ afont@iconcologia.net \\ N. Lainez \\ nuria.lainez.milagro@cfnavarra.es \\ S. Vázquez \\ sergio.vazquez.estevez@sergas.es
}

1 Medical Oncology Department, Hospital Álvaro CunqueiroComplexo Hospitalario Universitario de Vigo, Estrada Clara Campoamor, 34136312 Vigo, Spain

2 Medical Oncology Department, Parc Taulí Sabadell Hospital Universitari, Sabadell, Spain

3 Medical Oncology Department, Althaia, Xarxa Assisencial i Universitària de Manresa, Manresa, Spain

4 Medical Oncology Department, Hospital Universitario La Paz-Idipaz, Madrid, Spain

5 Medical Oncology Department, Hospital Universitario Son Espases, Palma de Mallorca, Spain

6 Medical Oncology Department, Hospital Universitario San Carlos, Madrid, Spain

7 Complexo Hospitalario Universitario de Ourense, Badalona, Spain

8 Medical Oncology Department, Germans Trias i Pujol University Hospital, Badalona, Spain

9 Medical Oncology Department, Complejo Hospitalario de Navarra, Pamplona, Spain

10 Medical Oncology Department, Hospital Universitario Lucus Augusti, Lugo, Spain 